\title{
Facile eco-friendly synthesis of novel chromeno[4,3-b]pyridine-2,5-diones and evaluation of their antimicrobial and antioxidant properties
}

\author{
SATYANARAYANA REDDY JAGGAVARAPU ${ }^{\mathrm{a}}$, ANAND SOLOMON KAMALAKARAN ${ }^{\mathrm{a}}$, \\ VENTKATA PRASAD JALLI ${ }^{\mathrm{a}}$, SRAVAN KUMAR GANGISETTY ${ }^{\mathrm{a}}$, MUNUSSWAMY \\ RAMANUJAM GANESH ${ }^{\mathrm{b}}$ and GOPIKRISHNA GADDAMANUGU ${ }^{\mathrm{a}, *}$ \\ ${ }^{a}$ Department of Chemistry, Sankar Foundation Research Institute, Naiduthota, Vishakapatnam 530 047, India \\ bISISM, SRM university, Kattangulathur, 603 203, India \\ e-mail: gkrishnagps@gmail.com
}

MS received 5 June 2013; accepted 13 November 2013

\begin{abstract}
Rapid and facileaccess to novel chromeno[4,3-b]pyridine-2,5-dione derivatives was achieved by a mild base catalysed reaction of 4-chloro-3-formylcoumarin and acetoacetamides in PEG-300 as recyclable solvent. The compounds were evaluated for their antimicrobial activities against 3 Gram-positive and 3 Gram-negative bacteria (Staphylococcus epidermis, Vibrio parahaemolyticus, Bacillus subtilis, Escherichia coli, Staphylococcus aureus and Klebsiella pneumonia) with Cefotaxime control. They were further subjected to antioxidant studies using DPPH test with ascorbic acid control. While compounds $\mathbf{5 d}$ and $\mathbf{5 k}$ showed promising broad spectrum antibacterial properties against all the evaluated bacteria, compound $\mathbf{5 g}$ exhibited good antioxidant properties.
\end{abstract}

Keywords. Chromeno[4,3-b]pyridine-2,5-diones; acetoacetamide; PEG-300; antimicrobial; antioxidant.

\section{Introduction}

Coumarins and 2-pyridones are classic heterocyclic scaffolds which constitute vital substructures of several natural products and received enormous admiration for their wide range of applications. Secondary metabolites and synthetic intermediates of 2pyridone scaffolds demonstrate broad spectrum of synthetic, material and biological applications. ${ }^{1}$ Ricinine $^{2}$ with its remarkable CNS stimulant activity was the first isolated 2-pyridone natural product followed by the discovery of analogous antibiotic natural products such as elfamycin, ${ }^{3}$ ilicolicin ${ }^{4}$ and efratomycin. ${ }^{5}$ With excellent vasodilating properties, ${ }^{6}$ synthetic 2 -pyridone analogues milrinone and amrinone are extensively used for the treatment of acute congestive heart failure. Similarly, L-697,661 was identified as a specific non-nucleoside reverse transcriptase inhibitor which demonstrates efficient anti-HIV properties. $^{7}$ 2Pyridone is an integral core of several alkaloids ${ }^{8}$ and is a key structural intermediate in the bacterial metabolism. ${ }^{9}$ Krawczyk et al. have developed 2-pyridones as novel class of multi-drug resistant modulators. ${ }^{10}$ On the other hand, coumarins ${ }^{11}$ exhibit excellent antioxidant, ${ }^{12}$ antibacterial, ${ }^{13}$ antirhinovirus, ${ }^{14}$

*For correspondence cytotoxic, ${ }^{15}$ anticancer, ${ }^{16}$ antimicrobial,${ }^{17}$ and antihypertensive properties. ${ }^{18}$ Aminocoumarins such as novobiocin, clorobiocin and coumermycin A1 with nitrogen functional group attached to the coumarin ring are elegant class of antibiotics which inhibit DNA gyrase enzyme involved in cell division of bacteria. ${ }^{19}$

Fused heterocyclic scaffolds with nitrogen and oxygen atoms are fundamental to the medicinal chemistry for the development of several new drugs. To date, very few reports are available in the literature regarding the synthesis of fused chromeno[4,3b]pyridine-2,5-dione scaffolds. One of the earliest documented report by Soliman et al. demonstrates synthesis of these scaffolds by the reaction of 4hydroxycoumarin and aminocrotonitrile. ${ }^{20}$ In a multistep protocol, Heber et al. reported the reaction of alkylaminocoumarin-3-carbaldehydes with Wittig ylides to achieve the required targets, albeit in poor yields. ${ }^{21}$ Ivanov et al. reported Erlenmeyer-Ploechl reaction of alkylaminocoumarin-3-carbaldehydes with $\mathrm{N}$-acetylgylcine derivatives to yield the chromeno[4,3b]pyridine-2,5-dione derivatives. ${ }^{22}$ However, the method suffers from the limitations of harsh reaction conditions of refluxing acetic acid and limited to the synthesis of only $\mathrm{N}$-alkyl derivatives in poor yields. Similarly, alternative synthesis of these fused systems reported by Kafka et al. using camphoranils with 
excess of dimethylmalonate suffer from poor yields. ${ }^{23}$ Owing to the necessity for an improved methodology for the synthesis of these fused scaffolds and prevalence of impressive biological properties of both pyridine-2ones and coumarins, we were interested in developing a mild synthetic protocol for the synthesis of the fused chromeno[4,3-b]pyridine-2,5-dione scaffolds.

\section{Experimental}

The solvents, bases and other general reagents were of AR grade and purchased from Otto Chemie. ${ }^{1} \mathrm{H}$ and ${ }^{13} \mathrm{C}$ NMR spectra are recorded on $400 \mathrm{MHz}$ Bruker Biospin FT-NMR spectrometer with $\mathrm{CDCl} 3$ as solvent and TMS as internal standard. Melting points were determined using NETZSCH DSC 200 instrument. IR spectra were recorded over Bruker a alphaT spectrophotometer. Mass spectral analysis was carried out using EIMStechniques. Elemental analysis was performed on Elementar Vario EL III CHNS analyzer. Analytical thin-layer chromatography (TLC) was performed on $0.2 \mathrm{~mm}$ precoated plate Kieselgel 60 F254 (Merck).

\subsection{General procedure for synthesis of acetoacetamides derivatives ( $3 \boldsymbol{a}-\boldsymbol{p})$}

To a stirred solution of amine $(0.01 \mathrm{~mol})$ in $10 \mathrm{ml}$ of PEG-300 was added to ethylacetoacatate $(0.03 \mathrm{~mol})$ in $100 \mathrm{ml}$ round bottom flask and refluxed at $120{ }^{\circ} \mathrm{C}$ for 1.5-2 h. After completion of the reaction (monitored by TLC) the reaction mixture was cooled and extracted with cold diethyl ether $(3 \times 10 \mathrm{~mL})$ and purified by column chromatography (10-25\% EtOAc in Hexane) gave the pure product $3 \mathbf{a}-\mathbf{p}$. Final products were confirmed with the reported literature. ${ }^{24}$

\subsection{General method for the synthesis of 3-acetyl-1- phenyl-1H-chromeno[4,3-b]pyridine-2,5-dione 5a-p}

To a solution of 4-chloro-3-formylcoumarin 1a (0.5 mmol) in PEG-300 (2 mL) was added acetoacetamides 3a $(0.5 \mathrm{mmol})$ and triethylamine $(0.5 \mathrm{mmol})$ then stirred at $25^{\circ} \mathrm{C}$ for $15 \mathrm{~min}$. The precipitate obtained was filtered by Whatman filter paper, washed with water and dried. Similar procedure was employed in case of substrates $\mathbf{5 b}-\mathbf{p}$.

2.2a 3-Acetyl-1-phenyl-1H-chromeno[4,3-b]pyridine2, 5-dione (5a): Whitesolid, $\mathrm{mp} 153-154^{\circ} \mathrm{C}$; [Found: $\mathrm{C}, 72.42 ; \mathrm{H}, 3.92 ; \mathrm{N}, 4.22 . \mathrm{C}_{20} \mathrm{H}_{13} \mathrm{NO}_{4}$ requires $\mathrm{C}$,
$72.50 ; \mathrm{H} 3.95 ; \mathrm{N}, 4.23 \%] ; v_{\max }(\mathrm{KBr}) 1719,1702$, 1679, 1618, 1583, 1479, 1418, 1240, $1041 \mathrm{~cm}^{-1}$; $\delta_{H}\left(400 \mathrm{MHz} \mathrm{CDCl}_{3}\right) 8.73(1 \mathrm{H}, \mathrm{s}), 7.72(1 \mathrm{H}, \mathrm{d}, J$ $6.8 \mathrm{~Hz}), 7.56-7.50(4 \mathrm{H}, \mathrm{m}), 7.44-7.43(2 \mathrm{H}, \mathrm{m}), 7.30$ $(1 \mathrm{H}, \mathrm{d}, J 6.8 \mathrm{~Hz}), 7.28-7.25(1 \mathrm{H}, \mathrm{m}), 2.71(3 \mathrm{H}, \mathrm{s})$; $\delta_{C}\left(100 \mathrm{MHz}, \mathrm{CDCl}_{3}\right) \quad \delta: 203.42,159.84,159.20$, $152.57,145.68,138.61,137.14,133.53,129.78$, 129.64,127.02, 126.07,125.98, 125.13, 118.77, 114.50, 103.35, 31.41, 8.57.; LCMS: $\mathrm{MH}^{+}, 332$.

2.2b 3-Acetyl-1-(4-fluorophenyl)-1H-chromeno[4,3-b] pyridine-2,5-dione $(\mathbf{5 b})$ : White solid, $\mathrm{mp} 134-135^{\circ} \mathrm{C}$; [Found: C, 68.70; $\mathrm{H}, 3.43 ; \mathrm{N}, 4.00 . \mathrm{C}_{20} \mathrm{H}_{12} \mathrm{FNO}_{4}$ requires $\mathrm{C}, 68.77 ; \mathrm{H}, 3.46 ; \mathrm{N}, 4.01 \%]$; $v_{\max }(\mathrm{KBr}) 1721$, 1705, 1680, 1621, 1579, 1469, 1421, 1245, $1048 \mathrm{~cm}^{-1}$; $\delta_{H}\left(400 \mathrm{MHz} \mathrm{CDCl}_{3}\right) 8.69(1 \mathrm{H}, \mathrm{s}), 7.70(1 \mathrm{H}, \mathrm{dd}, J$ $7.6 \mathrm{~Hz}, J 1.2 \mathrm{~Hz}), 7.58(1 \mathrm{H}, \mathrm{td}, J 7.6 \mathrm{~Hz}, J 1.2 \mathrm{~Hz})$, 7.49-7.40 (2H, m), $7.32(1 \mathrm{H}, \mathrm{dd}, J 6.8 \mathrm{~Hz}, J 0.8 \mathrm{~Hz})$, 7.30-7.20 (3H, m), $2.70(3 \mathrm{H}, \mathrm{s}) ; \delta_{C}\left(100 \mathrm{MHz} \mathrm{CDCl}_{3}\right)$ $203.25,163.87,161.87,159.83,159.10,152.54$, $145.52,137.22,134.49,133.63,128.17,127.01$, 125.97, 125.18, 118.80, 116.60, 114.41, 103.51, 31.39; LCMS: $\mathrm{MH}^{+}, 350$.

2.2c 3-Acetyl-1-(3-fluorophenyl)-1H-chromeno[4,3-b] pyridine-2,5-dione $(\mathbf{5 c})$ : White solid, $\mathrm{mp} 138-139{ }^{\circ} \mathrm{C}$; [Found: C, 68.70; H, 3.43; N, 4.00. $\mathrm{C}_{20} \mathrm{H}_{12} \mathrm{FNO}_{4}$ requires $\mathrm{C}, 68.77 ; \mathrm{H}, 3.46 ; \mathrm{N}, 4.01 \%]$; $v_{\max }(\mathrm{KBr}) 1721$, $1705,1683,1621,1587,1488,1421,1243,1021 \mathrm{~cm}^{-1}$; $\delta_{H}\left(400 \mathrm{MHz} \mathrm{CDCl}_{3}\right) 8.70(1 \mathrm{H}, \mathrm{s}), 7.71(1 \mathrm{H}, \mathrm{d}, J$ $6.8 \mathrm{~Hz}), 7.59-7.51(2 \mathrm{H}, \mathrm{m}), 7.31(1 \mathrm{H}, \mathrm{d}, J 6.8 \mathrm{~Hz})$, 7.30-7.26 (4H, m), $2.70(3 \mathrm{H}, \mathrm{s}) ; \delta_{C}\left(100 \mathrm{MHz} \mathrm{CDCl}_{3}\right)$ $203.18,163.59,161.60,159.58,159.03,152.54,145.28$, $139.66,139.58,137.23,133.69,131.05,130.98$, $\mathrm{m} 127.02$, 126.07, 125.20, 121.91, 118.80, 117.13, 116.96, 114.35, 114.33, 114.13, 103.65, 31.39; LCMS: $\mathrm{MH}^{+}, 350$.

2.2d 3-Acetyl-1-(4-bromophenyl)-1H-chromeno[4,3-b] pyridine-2,5-dione $(\mathbf{5 d})$ : White solid, $\mathrm{mp} 138-139^{\circ} \mathrm{C}$; [Found: $\mathrm{C}, 55.80 ; \mathrm{H}, 2.88 . \mathrm{C}_{18} \mathrm{H}_{11} \mathrm{BrO}_{5}$ requires $\mathrm{C}$, 55.84; H, 2.86\%]; $v_{\max }(\mathrm{KBr}) 1721,1705,1682,1621$, $1577,1472,1410,1233,1033 \mathrm{~cm}^{-1} ; \delta_{H}(400 \mathrm{MHz}$ $\left.\mathrm{CDCl}_{3}\right)$ 7.83-7.77 (2H, m), 7.59-7.51(2H, m), 7.45 $(1 \mathrm{H}, \mathrm{d}, J 8.8 \mathrm{~Hz}), 7.32(1 \mathrm{H}, \mathrm{t}, J 7.6 \mathrm{~Hz}), 7.21(1 \mathrm{H}$, d, $J 8.4 \mathrm{~Hz}), 6.80(1 \mathrm{H}, \mathrm{d}, J 8.8 \mathrm{~Hz}), 2.10(3 \mathrm{H}, \mathrm{s}) ; \delta_{C}$ $\left(100 \mathrm{MHz} \mathrm{CDCl}_{3}\right) 203.14,159.54,158.96,145.18$, $137.42,137.11,133.60,132.74,127.71,126.91$, 125.88, 125.13, 123.83, 118.71, 114.29, 103.55, 45.79, 31.33, 8.56; LCMS: $\mathrm{MH}^{+}, 411$. 
2.2e 3-Acetyl-1-(3-bromophenyl)-1H-chromeno[4,3-b] pyridine-2,5-dione $(\mathbf{5 e})$ : White solid, $\mathrm{mp} 132-133^{\circ} \mathrm{C}$; [Found: C, 58.56; H, 2.95, N, 3.41. $\mathrm{C}_{20} \mathrm{H}_{12} \mathrm{BrNO}_{4}$ requires $\mathrm{C}, 58.50 ; \mathrm{H}, 2.92, \mathrm{~N}, 3.41 \%]$; $v_{\max }(\mathrm{KBr}) 1723$, $1705,1683,1620,1533,1480,1420,1245,1033 \mathrm{~cm}^{-1}$; $\delta_{H}\left(400 \mathrm{MHz} \mathrm{CDCl}_{3}\right) 8.68(1 \mathrm{H}, \mathrm{s}), 7.76-7.70(3 \mathrm{H}, \mathrm{m})$, $7.57(1 \mathrm{H}, \mathrm{td}, J 6.8 \mathrm{~Hz}, 0.8 \mathrm{~Hz}), 7.49-7.38(3 \mathrm{H}, \mathrm{m})$, $7.33(1 \mathrm{H}, \mathrm{d}, J 6.8 \mathrm{~Hz}), 7.28(1 \mathrm{H}, \mathrm{dd}, J 6.8 \mathrm{~Hz}, 0.8 \mathrm{~Hz})$, $2.70(3 \mathrm{H}, \mathrm{s}) ; \delta_{C}\left(100 \mathrm{MHz} \mathrm{CDCl}_{3}\right)$ 203.09, 159.55, $158.58,152.51,145.20,139.48,137.22,133.67$, $132.95,130.82,129.37,127.00,126.01,125.18$, 124.90, 122.90, 118.77, 114.32, 103.64, 58.30, 31.37, 18.33; LCMS: $\mathrm{MH}^{+}, 411$.

2.2f 3-Acetyl-1-(4-chlorophenyl)-1H-chromeno[4,3-b] pyridine-2,5-dione $(\mathbf{5 f})$ : White solid, $\mathrm{mp} 130-131^{\circ} \mathrm{C}$; [Found: $\mathrm{C}, 65.61 ; \mathrm{H}, 3.28, \mathrm{~N}, 3.82 . \mathrm{C}_{19} \mathrm{H}_{14} \mathrm{O}_{5}$ requires C, 65.67; H, 3.31, N, 3.83\%]; $v_{\max }(\mathrm{KBr}) 1723,1706$, $1683,1630,1579,1481,1420,1238,1033 \mathrm{~cm}^{-1} ; \delta_{H}$ $\left(400 \mathrm{MHz} \mathrm{CDCl}_{3}\right) 8.67(1 \mathrm{H}, \mathrm{s}), 7.68(1 \mathrm{H}, \mathrm{d}, J 6.8 \mathrm{~Hz})$, $7.61-7.46(3 \mathrm{H}, \mathrm{m}), 7.40(1 \mathrm{H}, \mathrm{d}, J 7.2 \mathrm{~Hz}), 7.30(1 \mathrm{H}, \mathrm{d}$, $J 6.4 \mathrm{~Hz}), 7.25(1 \mathrm{H}, \mathrm{t}, \mathrm{J} 6.0 \mathrm{~Hz}), 2.68(3 \mathrm{H}, \mathrm{s}) ; \delta_{C}(100$ $\left.\mathrm{MHz} \mathrm{CDCl}_{3}\right)$ 203.18, 159.65, 159.02, 152.52, 145.28, $137.17,136.95,135.85,133.65,129.81,127.48$, $126.98,125.96,125.18,118.77,114.36,103.59,60.32$, 31.38, 14.13; LCMS: $\mathrm{MH}^{+}, 366$.

$2.2 \mathrm{~g}$ 3-Acetyl-1-p-tolyl-1H-chromeno[4,3-b]pyridine2,5-dione (5g): White solid, $\mathrm{mp} 132-133^{\circ} \mathrm{C}$; [Found: C, 73.03; H, 4.38, N, $4.06 \mathrm{C}_{20} \mathrm{H}_{16} \mathrm{O}_{6}$ requires C, 72.96; $\mathrm{H}, 4.38, \mathrm{~N}, 4.05 \%$ ]; $v_{\max }(\mathrm{KBr}) 1722,1705,1683,1621$, $1579,1480,1420,1230,1039 \mathrm{~cm}^{-1} ; \delta_{H}(400 \mathrm{MHz}$ $\left.\mathrm{CDCl}_{3}\right) 8.70(1 \mathrm{H}, \mathrm{s}), 7.70(1 \mathrm{H}, \mathrm{d}, J 6.4 \mathrm{~Hz}), 7.55(1 \mathrm{H}, \mathrm{t}$, $J 5.6 \mathrm{~Hz}), 7.33-7.23(6 \mathrm{H}, \mathrm{m}), 2.69(3 \mathrm{H}, \mathrm{s}), 2.42(3 \mathrm{H}, \mathrm{s})$; $\delta_{C}\left(100 \mathrm{MHz} \mathrm{CDCl}_{3}\right) 203.45,159.86,159.15,152.44$, $145.75,139.91,136.92,136.02,133.37,130.07$, 126.90, 125.69, 125.00, 118.64, 114.44, 103.10, 31.33, 21.08, 18.26; LCMS: $\mathrm{MH}^{+}, 346$.

2.2h 3-Acetyl-1-m-tolyl-1H-chromeno[4,3-b]pyridine2,5-dione $(\mathbf{5 h})$ : White solid, mp $138-139^{\circ} \mathrm{C}$; [Found: C, 73.03; $\mathrm{H}, 4.38, \mathrm{~N}, 4.06 \mathrm{C}_{20} \mathrm{H}_{16} \mathrm{O}_{6}$ requires C, 72.96; $\mathrm{H}, 4.38, \mathrm{~N}, 4.05 \%$ ]; $v_{\max }(\mathrm{KBr}) 1721,1705,1682,1623$, 1579, 1482, 1420, 1241, $1039 \mathrm{~cm}^{-1} ; \delta_{H}(400 \mathrm{MHz}$ $\left.\left.\mathrm{CDCl}_{3}\right) 8.70(1 \mathrm{H}, \mathrm{s})\right), 7.70(1 \mathrm{H}, \mathrm{s}, \mathrm{J} 6.4 \mathrm{~Hz}), 7.55(1 \mathrm{H}$, $\mathrm{t}, J 5.6 \mathrm{~Hz}), 7.33-7.23(6 \mathrm{H}, \mathrm{m}), 2.69(3 \mathrm{H}, \mathrm{s}), 2.42$ $(3 \mathrm{H}, \mathrm{s}) ; \delta_{C}\left(100 \mathrm{MHz} \mathrm{CDCl}_{3}\right) 203.51,159.95,159.30$, $152.63,145.79,139.99,138.58,137.15,133.53$, $130.59,129.48,127.07,126.62,125.99,125.16$, 123.07, 118.82, 114.58, 103.27, 31.46, 21.30; LCMS: $\mathrm{MH}^{+}, 346$. 2.2i 3-Acetyl-1-(4-methoxyphenyl)-1H-chromeno[4, 3-b]pyridine-2,5-dione (5i): White solid, mp 230$232^{\circ} \mathrm{C}$; [Found: $\mathrm{C}, 58.22 ; \mathrm{H}, 2.69 ; \mathrm{N}, 3.75 . \mathrm{C}_{21} \mathrm{H}_{15} \mathrm{NO}_{5}$ requires $\mathrm{C}, 58.23 ; \mathrm{H}, 2.71, \mathrm{~N}, 3.77 \%]$; $v_{\max }(\mathrm{KBr}) 1722$, $1708,1683,1620,1579,1480,1420,1239,1039 \mathrm{~cm}^{-1}$; $\delta_{H}\left(400 \mathrm{MHz} \mathrm{CDCl}_{3}\right) 8.70(1 \mathrm{H}, \mathrm{s}), 7.70(1 \mathrm{H}, \mathrm{d}, J$ $6.8 \mathrm{~Hz}), 7.55(1 \mathrm{H}, \mathrm{t}, J 6.8 \mathrm{~Hz}), 7.35-7.22(4 \mathrm{H}, \mathrm{m})$, $7.02(1 \mathrm{H}, \mathrm{d}, J 7.2 \mathrm{~Hz}), 3.86(3 \mathrm{H}, \mathrm{s}), 2.70(3 \mathrm{H}, \mathrm{s}) ; \delta_{C}$ $\left.\left(100 \mathrm{MHz} \mathrm{CDCl}_{3}\right)\right)$ 203.50, 160.30, 160.02, 159.22, $152.51,145.87,136.97,133.42,131.29,127.20$, 125.06, 118.70, 114.70, 103.13, 55,59, 31.38; LCMS: $\mathrm{MH}^{+}, 362$.

\section{$2.2 \mathrm{j}$ 3-Acetyl-1-(3-methoxyphenyl)-1H-chromeno}

[4,3-b]pyridine-2,5-dione (5j): White solid, mp 133$135^{\circ} \mathrm{C}$; [Found: $\mathrm{C}, 69.73 ; \mathrm{H}, 4.15 ; \mathrm{N}, 3.87 . \mathrm{C}_{21} \mathrm{H}_{15} \mathrm{NO}_{5}$ requires $\mathrm{C}, 69.80 ; \mathrm{H}, 4.18, \mathrm{~N}, 3.88 \%]$; $v_{\max }(\mathrm{KBr}) 1723$, $1707,1688,1620,1579,1481,1420,1240,1041 \mathrm{~cm}^{-1}$; $\delta_{H}\left(400 \mathrm{MHz} \mathrm{CDCl}_{3}\right) 8.71(1 \mathrm{H}, \mathrm{s}), 7.70(1 \mathrm{H}, \mathrm{d}, J$ $6.4 \mathrm{~Hz}), 7.56(1 \mathrm{H}, \mathrm{t}, J 6.4 \mathrm{~Hz}), 7.44(1 \mathrm{H}, \mathrm{t}, J 6.4 \mathrm{~Hz})$, $7.31(1 \mathrm{H}, \mathrm{d}, J 6.4 \mathrm{~Hz}), 7.39-7.21(2 \mathrm{H}, \mathrm{m}), 7.04(1 \mathrm{H}$, d, J $6.8 \mathrm{~Hz}), 7.00-6.96(2 \mathrm{H}, \mathrm{m}), 3.85(3 \mathrm{H}, \mathrm{s}), 2.71$ $(3 \mathrm{H}, \mathrm{s}) ; \delta_{C}\left(100 \mathrm{MHz} \mathrm{CDCl}_{3}\right) 203.44,160.33,159.15$, $152.53,145.70,139.57,137.05,133.50,130.40$, $126.98,125.93,125.10,118.09,115.76,114.47$, 111.87, 103.22, 55.58, 31.40; LCMS: $\mathrm{MH}^{+}, 362$.

2.2k 3-Acetyl-1-(3-nitrophenyl)-1H-chromeno[4,3-b] pyridine-2,5-dione $(\mathbf{5 k})$ : White solid, $\mathrm{mp} 134-136^{\circ} \mathrm{C}$; [Found: C, 63.77; H, 3.28; N, 7.44. $\mathrm{C}_{20} \mathrm{H}_{12} \mathrm{~N}_{2} \mathrm{O}_{6}$ requires $\mathrm{C}, 63.83 ; \mathrm{H}, 3.21, \mathrm{~N}, 7.44 \%]$; $v_{\max }(\mathrm{KBr}) 1725$, 1706, 1681, 1620, 1579, 1477, 1420, 1238, $1038 \mathrm{~cm}^{-1}$; $\delta_{H}\left(400 \mathrm{MHz} \mathrm{CDCl}_{3}\right) 8.71(1 \mathrm{H}, \mathrm{s}), 8.42-8.31(2 \mathrm{H}, \mathrm{m})$, $7.83(1 \mathrm{H}, \mathrm{d}, J 6.4 \mathrm{~Hz}), 7.77(1 \mathrm{H}, \mathrm{t}, J 6.4 \mathrm{~Hz}), 7.70$ $(1 \mathrm{H}, \mathrm{d}, J 6.4 \mathrm{~Hz}),, 7.59(1 \mathrm{H}, \mathrm{t}, J 6.0 \mathrm{~Hz}), 7.33(1 \mathrm{H}$, d, $J 6.8 \mathrm{~Hz}), 7.28(1 \mathrm{H}, \mathrm{t}, J 6.0 \mathrm{~Hz}), 2.71(3 \mathrm{H}, \mathrm{s}) ; \delta_{C}$ $\left(100 \mathrm{MHz} \mathrm{CDCl}_{3}\right)$ 202.81, 159.50, 158.86, 152.64, $148.75,144.65,139.29,137.65,134.02,132.32$, $130.69,127.16,126.30,125.39,124.65,121.87$, 118.96, 114.27, 104.34, 31.44; LCMS: $\mathrm{MH}^{+}, 377$.

2.21 3-Acetyl-1-(2-nitrophenyl)-1H-chromeno[4,3-b] pyridine-2,5-dione $(\mathbf{5 l})$ : White solid, $\mathrm{mp} 138-140^{\circ} \mathrm{C}$; [Found: $\mathrm{C}, 62.12 ; \mathrm{H}, 3.54 ; \mathrm{N}, 3.81 . \mathrm{C}_{19} \mathrm{H}_{13} \mathrm{NO}_{7}$ requires $\mathrm{C}, 62.13 ; \mathrm{H}, 3.57, \mathrm{~N}, 3.81 \%]$; $v_{\max }(\mathrm{KBr}) 1723,1704$, 1682, 1620, 1579, 1482, 1420, 1218, $1045 \mathrm{~cm}^{-1}$; $\delta_{H}\left(400 \mathrm{MHz} \mathrm{CDCl}_{3}\right) 8.69(1 \mathrm{H}, \mathrm{s}), 8.23(1 \mathrm{H}, \mathrm{d}, J$ $6.4 \mathrm{~Hz}), 8.12(1 \mathrm{H}, \mathrm{d}, J 6.8 \mathrm{~Hz}), 7.82-7.71(2 \mathrm{H}, \mathrm{m})$, $7.77(1 \mathrm{H}, \mathrm{t}, J 6.4 \mathrm{~Hz}), 7.70(1 \mathrm{H}, \mathrm{t}, J 6.4 \mathrm{~Hz}),$, $(1 \mathrm{H}, \mathrm{t}, J 6.0 \mathrm{~Hz}), 7.33-722(2 \mathrm{H}, \mathrm{m}), 2.69(3 \mathrm{H}, \mathrm{s}) ; \delta_{C}$ $\left.(100 \mathrm{MHz} \mathrm{CDCl})_{3}\right) 160.12,158.50,152.57,151.22$, 
$144.21,137.57,132.38,125.30,125.01,124.01$, 120.32, 119.18, 118.12, 117.57, 103.77, 98.56, 91.40, 23.56, 21.07; LCMS: $\mathrm{MH}^{+}, 377$.

\section{2m 3-Acetyl-1-(pyridin-2-yl)-1H-chromeno[4,3-b]} pyridine-2,5-dione $(\mathbf{5 m})$ : white solid, $\mathrm{mp} 132-135^{\circ} \mathrm{C}$; [Found: $\mathrm{C}, 68.61 ; \mathrm{H}, 3.61 \mathrm{~N}, 8.42 . \mathrm{C}_{19} \mathrm{H}_{12} \mathrm{~N}_{2} \mathrm{O}_{4}$ requires $68.67 ; \mathrm{H}, 3.64 \mathrm{~N}, 8.43 \%$ ]; $v_{\max }(\mathrm{KBr}) 1724$, $1707,1682,1621,1581,1481,1421,1239,1040 \mathrm{~cm}^{-1}$; $\delta_{H}\left(400 \mathrm{MHz} \mathrm{CDCl}_{3}\right) \quad \delta 9.04(1 \mathrm{H}, \mathrm{s}), 8.69(1 \mathrm{H}, \mathrm{s})$, $8.08(1 \mathrm{H}, t, J 6.0 \mathrm{~Hz}), 7.93(1 \mathrm{H}, \mathrm{d}, J 5.4 \mathrm{~Hz}), 7.69$ $7.59(3 \mathrm{H}, \mathrm{m}), 7.41-7.36(2 \mathrm{H}, \mathrm{m}), 2.64(3 \mathrm{H}, \mathrm{s}) ; \delta_{C}$ $\left(100 \mathrm{MHz} \mathrm{CDCl}_{3}\right)$ 188.30, 186.10, 165.55, 161.27, $159.09,154.79,135.93,135.47,129.64,125.86$, 125.72 , 125.45, 124.67, 117.46, 116.49, 114.45, 110.17; LCMS: $\mathrm{MH}^{+}, 333$.

\section{2n 3-Acetyl-1-butyl-1H-chromeno[4,3-b]pyridine-}

2,5-dione (5n): White solid, $\mathrm{mp} 138-141^{\circ} \mathrm{C}$; [Found: C, 69.37; $\mathrm{H}, 5.46, \mathrm{~N}, 4.49 . \mathrm{C}_{24} \mathrm{H}_{16} \mathrm{O}_{5}$ requires $\mathrm{C}, 69.44$; $\mathrm{H}, 5.50, \mathrm{~N}, 4.50 \%]$; $v_{\max }(\mathrm{KBr}) 1721,1707,1681,1620$, $1579,1460,1421,1219,1039 \mathrm{~cm}^{-1} ; \delta_{H}(400 \mathrm{MHz}$ $\left.\mathrm{CDCl}_{3}\right) 8.62(1 \mathrm{H}, \mathrm{s}), 7.66(1 \mathrm{H}, \mathrm{d}, J 6.0 \mathrm{~Hz}), 7.52(1 \mathrm{H}$, t, $J 6.8 \mathrm{~Hz}), 7.29(1 \mathrm{H}, \mathrm{d}, J 6.4 \mathrm{~Hz}), 7.26-7.21(1 \mathrm{H}, \mathrm{m})$, $4.08(1 \mathrm{H}, \mathrm{t}, J 6.0 \mathrm{~Hz}), 2.67(3 \mathrm{H}, \mathrm{s}), 1.82(2 \mathrm{H}, \mathrm{q}),$, $(\mathrm{m}, 2 \mathrm{H}), 0.98(3 \mathrm{H}, \mathrm{t}) ; \delta_{C}\left(100 \mathrm{MHz} \mathrm{CDCl}_{3}\right) 203.75$, $159.98,159.31,152.43,145.34,136.63,133.20$, $126.81,125.13,124.97,118.64,114.58,102.67,50.73$, 31.42, 31.03, 19.76, 13.48; LCMS: $\mathrm{MH}^{+}, 312$.

2.2o 3-Acetyl-1-ethyl-1H-chromeno[4,3-b]pyridine2,5-dione (5o): White solid, mp 132-134 ${ }^{\circ} \mathrm{C}$; [Found: $\mathrm{C}, 67.77 ; \mathrm{H}, 4.58, \mathrm{~N}, 4.94, \mathrm{C}_{16} \mathrm{H}_{13} \mathrm{NO}_{4}$ requires $\mathrm{C}, 67.84 ; \mathrm{H}, 4.63,4.94 \%]$; $v_{\max }(\mathrm{KBr}) 1725,1708$, $1679,1621,1592,1479,1456,1246,1050 \mathrm{~cm}^{-1} ; \delta_{H}$ $\left(400 \mathrm{MHz} \mathrm{CDCl}_{3}\right) 8.67(1 \mathrm{H}, \mathrm{s}), 7.67(1 \mathrm{H}, \mathrm{dd}, J 6.4 \mathrm{~Hz}$, $0.8 \mathrm{~Hz}), 7.54(1 \mathrm{H}, \mathrm{td}, J 6.8, J 1.2 \mathrm{~Hz}), 7.31(1 \mathrm{H}, \mathrm{dd}, J$ $6.8 \mathrm{~Hz}, J 0.8 \mathrm{~Hz}), 7.24(1 \mathrm{H}, \mathrm{td}, J 6.8, J 0.8 \mathrm{~Hz}), 4.16$ $(2 \mathrm{H}, \mathrm{q}, J 5.6 \mathrm{~Hz}), 2.68(3 \mathrm{H}, \mathrm{s}), 1.47(3 \mathrm{H}, \mathrm{t}, J 5.6 \mathrm{~Hz})$; $\delta_{C}\left(100 \mathrm{MHz} \mathrm{CDCl}_{3}\right) 203.77,159.86,159.29,152.42$, $144.97,136.66,133.21,126.80,125.13,124.98$, $118.64,114.57,102.85,46.05,31.45,14.39,8.54$; LCMS: $\mathrm{MH}^{+}, 284$.

2.2p 3-Acetyl-1-benzyl-1H-chromeno[4,3-b]pyridine2,5-dione (5p): White solid, $\mathrm{mp} 135-137^{\circ} \mathrm{C}$; [Found: $\mathrm{C}, 72.97 ; \mathrm{H}, 4.34 ; \mathrm{N}, 4.05 \mathrm{C}_{21} \mathrm{H}_{15} \mathrm{NO}_{4}$ requires $\mathrm{C}$, $73.03 ; \mathrm{H}, 4.38, \mathrm{~N}, 4.06 \%]$; $v_{\max }(\mathrm{KBr}) 1723,1705$, $1681,1621,1592,1489,1458,1247,1053 \mathrm{~cm}^{-1} ; \delta_{H}$ $\left(400 \mathrm{MHz} \mathrm{CDCl}_{3}\right) 8.63(1 \mathrm{H}, \mathrm{s}), 7.66(1 \mathrm{H}, \mathrm{d}, J 6.0 \mathrm{~Hz})$, $7.52(1 \mathrm{H}, \mathrm{t}, J 6.0 \mathrm{~Hz}), 7.44-7.31(5 \mathrm{H}, \mathrm{m}), 7.30-7.19$
$(2 \mathrm{H}, \mathrm{m}), 5.24(2 \mathrm{H}, \mathrm{s}), 2.68(3 \mathrm{H}, \mathrm{s}) ; \delta_{C}(100 \mathrm{MHz}$ $\left.\mathrm{CDCl}_{3}\right) \quad 203.62,160.09,159.15, \quad 152.46,145.08$, $136.77,134.13,133.34,129.27,128.96,128.73$, $126.88,125.33,125.04,118.67,114.54,103.13,53.22$, 31.47; LCMS: $\mathrm{MH}^{+}, 346$.

\section{Results and discussions}

Application of green and sustainable chemistry protocols has seen enormous surge in recent times for the development of novel and eco-friendly methodologies towards the synthesis of valuable synthetic scaffolds and drug intermediates. Polyethylene glycol (PEG) has gained wide popularity as alternative solvent in contributing to such green methodologies by successfully plummeting the generation of industrial waste. ${ }^{25}$

We have earlier demonstrated the utility of 4-chloro3-formylcoumarin as a versatile starting material for the syntheses of various privileged heterocycles such as chromeno-quinolines, ${ }^{26 \mathrm{a}}$ chromeno-benzazepines, ${ }^{26 \mathrm{~b}}$ chromeno-pyrimidine-N-oxides ${ }^{26 \mathrm{c}}$ and chromenotrioxabicylco[3,3,1]nonadienes. ${ }^{26 \mathrm{~d}}$ In continuation of our efforts to synthesize novel heterocyclic molecules of biological importance, we envisaged a mild base mediated synthesis of the target molecules from 4chloro-3-formylcoumarin and acetoacetamides via simultaneous nucleophilic $N$-alkylation and Knoevenagel reactions using PEG-300 as a recyclable eco-friendly solvent.

The popular methodologies for the synthesis of acetoacetamides in general employ reaction of amines with excess of ethyl acetoacetate in solvents such as benzene, toluene or xylenes at reaction temperatures up to $140^{\circ} \mathrm{C}{ }^{24}$ As an alternative eco-friendly procedure for the synthesis of starting materials $\mathbf{3 a}-\mathbf{p}$, we explored the possibility of PEG-300 as a benign solvent for their reaction. We found that there are no reports utilizing PEG-300 as a solvent for the synthesis of the acetoacetamides to date. When the reaction of aniline 1a was conducted in PEG-300 with 1 equivalent of ethyl acetoacetate, the product $\mathbf{3 a}$ was isolated in moderate yields $(60 \%)$ after $2 \mathrm{~h}$ of reaction at $120^{\circ} \mathrm{C}$ (table 1 , entry 1 ). Increasing the quantity of ethyl acetoacetate to 2 equivalents resulted in good yields $(81 \%)$ of the required acetoacetamide 3a (table 1, entry 2). The present optimized reaction conditions successfully circumvented the utilization of large excess of ethyl acetoacetate unlike the earlier reported methodologies. As shown in table 1 , the reaction conditions were further exploited for 
Table 1. Synthesis of acetoacetamides with diverse amines and ethyl acetoacetate ${ }^{\mathrm{a}}$.

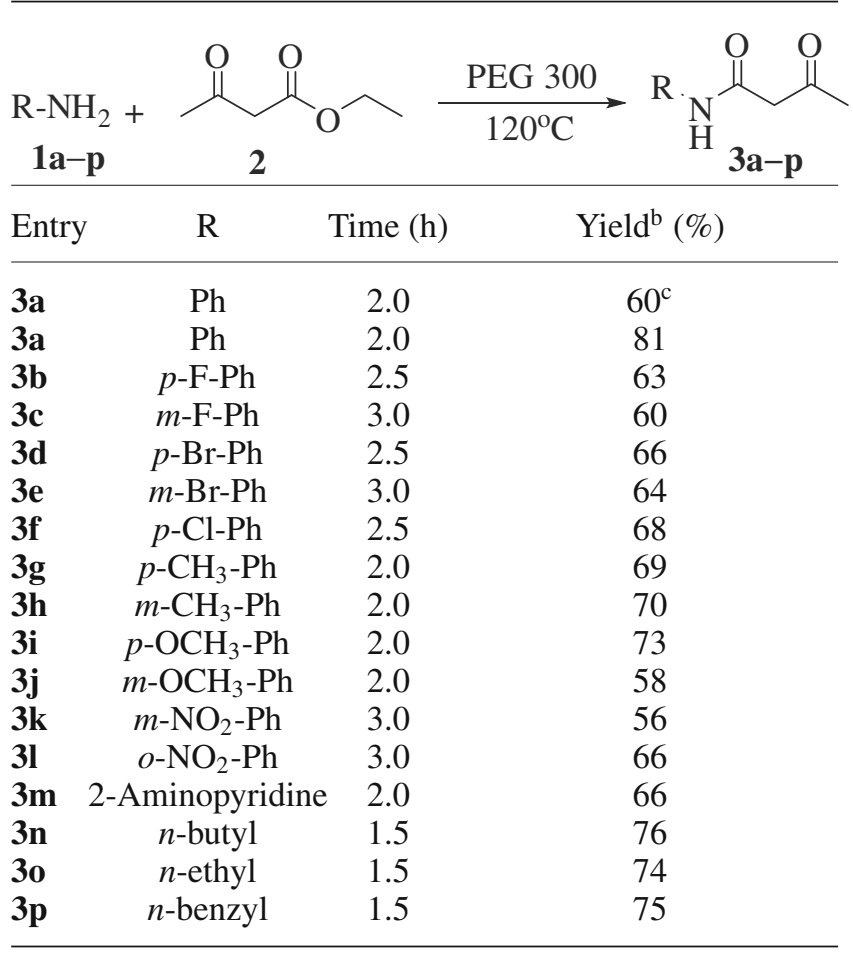

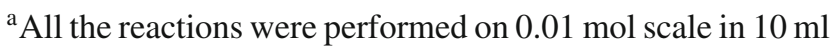
solvent at $120^{\circ} \mathrm{C}$

${ }^{\mathrm{b}}$ Isolated yields

${ }^{\mathrm{c}}$ One equivalent of ethyl acetoacetate

the synthesis of acetoacetamides $\mathbf{3 b}-\mathbf{p}$ (entries 216) successfully with a variety of functional groups in good yields (56-81\%). The methodology worked satisfactorily for aryl, heteroaryl and aliphatic substrates.

To further optimize the reaction conditions for the synthesis of chromeno[4,3-b]pyridine-2,5-diones 5ap, 4-choloro-3-formylcoumarin $\mathbf{4}$ and acetoacetanilide 3a were chosen as model substrates. An initial reaction was attempted where $\mathbf{4}$ was reacted with acetoacetamide 3a in PEG-300 employing triethylamine base. Completion of the reaction was indicated by the formation of a precipitate which could be easily filtered from the reaction mixture. To our satisfaction, we found that the product obtained was in good yield $(83 \%)$ and of high purity (table 2 , entry 1$)$. To ascertain the efficiency of triethylamine, various bases as shown in table 2 were screened for the reaction. While bases such as $\mathrm{K}_{2} \mathrm{CO}_{3}$ and $\mathrm{NaHCO}_{3}$ gave moderate yields (50-60\%) of product 5a after $6 \mathrm{~h}$ of reaction, surprisingly, sodium acetate and DMAP did not catalyse the reaction (table 2, entries 2-5). Competitive yields of 5a were observed when DMAP (78\%) and DBU (70\%) were employed as bases (table 2, entries 6 and 7). Triethylamine was considered as the
Table 2. Screening studies of reaction of acetoacetamide (3a) and 4-chloro-3-formylcoumarin (4) with various bases in PEG-300 ${ }^{\mathrm{a}}$.

\begin{tabular}{|c|c|c|c|}
\hline 3a & + & $\frac{P}{B}$ & R \\
\hline Entry & Base & Time & Yield $^{\mathrm{c}}(\%)$ \\
\hline 1 & $\mathrm{Et}_{3} \mathrm{~N}$ & $15 \mathrm{~min}$ & $83^{b}$ \\
\hline 2 & $\mathrm{~K}_{2} \mathrm{CO}_{3}$ & $6 \mathrm{~h}$ & 50 \\
\hline 3 & $\mathrm{NaHCO}_{3}$ & $6 \mathrm{~h}$ & 60 \\
\hline 4 & $\mathrm{NaOAc}$ & $10 \mathrm{~h}$ & $\mathrm{NR}^{\mathrm{d}}$ \\
\hline 5 & DMAP & $10 \mathrm{~h}$ & NR \\
\hline 6 & DIEA & $15 \mathrm{~min}$ & 78 \\
\hline 7 & DBU & $15 \mathrm{~min}$ & 70 \\
\hline
\end{tabular}

${ }^{a}$ All the reactions were performed on $0.5 \mathrm{mmol}$ scale with 1 equiv of base in $2 \mathrm{ml}$ solvent at room temperature

${ }^{\mathrm{b}}$ Pure precipitated yield

${ }^{\mathrm{c}}$ Isolated yields

${ }^{\mathrm{d}}$ No reaction.

best base from the standardization of reaction conditions. PEG solvent could easily be recycled by washing with small quantities $(5 \mathrm{ml})$ of diethyl ether. Our attempts to synthesize the target chromeno[4,3b]pyridine-2,5-dione $\mathbf{3 a}$ in one-pot procedure starting from aniline 1a unfortunately did not yield satisfactory results.

To validate general feasibility of the methodology, variety of aromatic and aliphatic acetoacetamides $\mathbf{3 b}-\mathbf{p}$ were utilized in the reaction with 4-chloro-3formylcoumarin under the optimized reaction conditions. As demonstrated in table 3, halogen substituted acetoacetamides $\mathbf{3 b}-\mathbf{f}$ possessing $p$-fluoro, $m$-flouro, $p$-bromo, $m$-bromo and $p$-chloro substituents reacted smoothly to afford the corresponding benzopyrano-2pyridoneproducts $\mathbf{5 b}-\mathbf{f}$ in $78-82 \%$ yields. While electron donating substrates $\mathbf{3 g}-\mathbf{j}$ possessing methyl and methoxy substituents afforded slightly higher yields (84-90\%) of the corresponding products $5 \mathbf{g}-\mathbf{j}$, the electron withdrawing substrates $p-\mathrm{NO}_{2}$ and $m-\mathrm{NO}_{2}$ groups afforded 72 and $75 \%$ yields of products $\mathbf{5 k}$ and 5l, respectively. Heteroaromatic and aliphatic substrates $3 \mathbf{m}-\mathbf{p}$ also furnished good yields (72-84\%) of coumarino[4,3-b]pyrid-2-one products $\mathbf{5 m}-\mathbf{p}$ under the optimized reaction conditions. The present recyclable eco-friendly methodology offers advantages of the obtaining pure precipitates of $\mathbf{5 a}-\mathbf{p}$ in good to excellent yields without requirement of tedious purification protocols. 
Table 3. Synthesis of chromeno [4, 3-b] pyridine-2, 5-diones.

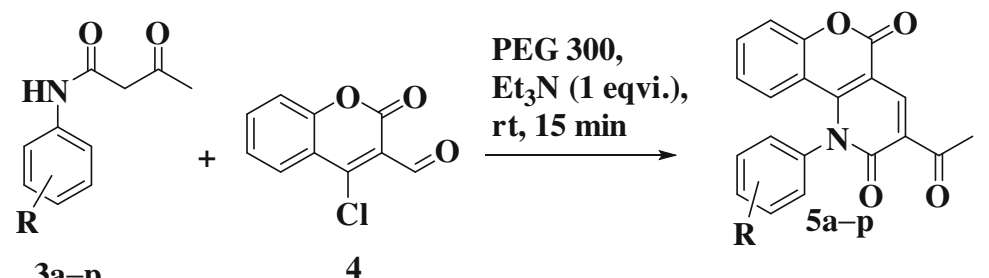

1<smiles>CC(=O)CC(=O)Nc1ccccc1</smiles>

3a

2<smiles>CC(=O)CC(=O)Nc1ccc(F)cc1</smiles>

3<smiles>CC(=O)CC(=O)Nc1ccc(Cl)cc1</smiles>

4<smiles>CC(=O)CC(=O)Nc1ccc(Br)cc1</smiles>

5<smiles>CC(=O)CC(=O)Nc1ccc(C)cc1</smiles>

6<smiles>CC(=O)CC(=O)Nc1cccc(C)c1</smiles>

7<smiles>CC(=O)CC(=O)Nc1cccc(F)c1</smiles>

8<smiles>CC(=O)CC(=O)Nc1cccc(Br)c1</smiles><smiles>CC(=O)c1cc2c(=O)oc3ccccc3c2n(-c2ccccc2)c1=O</smiles> 
Table 3. (continued)

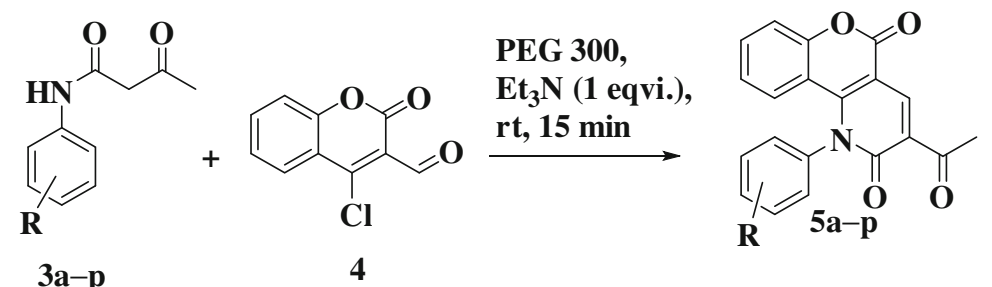

\begin{tabular}{|c|c|c|c|}
\hline Entry & Acetoacetamide & Chromeno[4,3-b]pyridine-2,5-dione (5a-n) & Yield $(\%)^{\mathrm{a}}$ \\
\hline 9 & & & 83 \\
\hline 10 & & & 85 \\
\hline 11 & & & 75 \\
\hline 12 & & & 71 \\
\hline 13 & & & 72 \\
\hline 14 & & & 84 \\
\hline 15 & & & 83 \\
\hline 16 & & & 80 \\
\hline
\end{tabular}

${ }^{a}$ Yields refer to the precipitated products 
Table 4. Evaluation of antibacterial and antioxidant properties for $\mathbf{5 a}-\mathbf{p}$.

\begin{tabular}{|c|c|c|c|c|c|c|c|}
\hline Entry & E. coli (-ve) & $\begin{array}{c}\text { V. parahaemolyticus } \\
(-\mathrm{ve})\end{array}$ & $\begin{array}{c}\text { K. pneumoniae } \\
(-\mathrm{ve})\end{array}$ & $\begin{array}{l}\text { S. epidermidis } \\
(+\mathrm{ve})\end{array}$ & $\begin{array}{l}\text { B. subtilis } \\
\text { (+ve) }\end{array}$ & $\begin{array}{l}\text { S. aureus } \\
(+\mathrm{ve})\end{array}$ & $\begin{array}{c}\text { DPPH inhibition } \\
(\%)\end{array}$ \\
\hline $5 \mathbf{a}$ & $>200$ & $>200$ & $>200$ & $>200$ & $>200$ & $>200$ & 80.21 \\
\hline $5 \mathbf{b}$ & $>200$ & $>200$ & $>200$ & $>200$ & $>200$ & $>200$ & 26.23 \\
\hline $5 c$ & $>200$ & $>200$ & $>200$ & $>200$ & $>200$ & $>200$ & 42.41 \\
\hline $5 d$ & 6.25 & 12.5 & 12.5 & 25 & 12.5 & 3.13 & 39.50 \\
\hline $5 e$ & $>200$ & $>200$ & $>200$ & $>200$ & $>200$ & $>200$ & 55.48 \\
\hline $5 f$ & $>200$ & $>200$ & $>200$ & $>200$ & $>200$ & $>200$ & NA \\
\hline $5 g$ & $>200$ & $>200$ & $>200$ & $>200$ & $>200$ & $>200$ & 62.13 \\
\hline $5 h$ & $>200$ & $>200$ & $>200$ & $>200$ & $>200$ & $>200$ & NA \\
\hline $5 \mathbf{i}$ & $>200$ & $>200$ & $>200$ & $>200$ & $>200$ & $>200$ & 36.27 \\
\hline $5 \mathbf{j}$ & 12.5 & 50 & 50 & 50 & 25 & $\mathbf{5 0}$ & 16.39 \\
\hline $5 \mathbf{k}$ & 12.5 & 6.25 & 12.5 & 3.13 & 6.25 & 3.13 & 49.34 \\
\hline 51 & $>200$ & $>200$ & $>200$ & $>200$ & $>200$ & $>200$ & NA \\
\hline $5 \mathrm{~m}$ & $>200$ & $>200$ & $>200$ & $>200$ & $>200$ & $>200$ & 29.44 \\
\hline
\end{tabular}

For evaluation of antibacterial properties of the synthesized compounds 5a-p (table 4), clinically active Gram-negative Escherichia coli, Vibrio parahaemolyticus, Klebsiella pneumonia and Gram-positive Staphylococcus epidermidis, Bacillus subtilis, and Staphylococcus aureus bacteria were selected. Dimethyl sulfoxide was chosen as negative control while Cefotoxime was chosen as positive control due to its broad spectrum antibacterial activity against both Gram-positive and Gram-negative bacteria. The concentration at which the compounds inhibited the visible growth of the microbial cultures was taken as MIC for that compound by using the standard protocol of NCCLS Broth Microdilution MIC method. ${ }^{27}$ The MIC values listed in table 4 suggest that compounds $\mathbf{5 d}, \mathbf{5 j}, \mathbf{5 k}, \mathbf{5 n}$ and $\mathbf{5 o}$ showed promising broad spectrum antibacterial activities. More specifically, compounds $\mathbf{5 d}$ and $\mathbf{5 k}$ demonstrated maximum activity, where $\mathbf{5 d}$ was more selective in inhibiting Staphylococcus aureus $(3.13 \mu \mathrm{g} / \mathrm{mL})$ and $\mathbf{5 k}$ exhibits maximum activity against both Staphylococcus aureus and Staphylococcus epidermidis $(3.13 \mu \mathrm{g} / \mathrm{mL})$.

The evaluation of total antioxidant activities of the compounds $\mathbf{5 a}-\mathbf{p}$ were evaluated using free radical scavenging activity determined by 1,1-diphenyl picryl hydrzyl (DPPH) procedure..$^{28}$ The inhibition percentage equation of the radical scavenging the radical scavenging activity was calculated by using the equation

$$
\text { Inhibition }(\%)=\left[\left(A_{0}-A_{\mathrm{S}}\right) / \mathrm{A}_{0}\right] \times 100,
$$

where $A_{0}$ is absorbance of the blank and $A_{\mathrm{S}}$ is absorbance of the sample at $515 \mathrm{~nm}$. All assays were conducted in triplicate. Testing was performed with $180 \mu \mathrm{L}$ of $0.004 \%$ methanolic solution of DPPH pipetted into each well of a 96-well plate followed by $20 \mu \mathrm{L}$ $(20 \mathrm{mg} / \mathrm{ml})$ of sample or solvent for the blank. Ascorbic acid was used as the positive control. The mixture was incubated at $30^{\circ} \mathrm{C}$ for $1 \mathrm{~h}$, and the absorbance at $515 \mathrm{~nm}$ was measured with a microplate reader. From the results of the screening studies displayed in table 4, it can be suggested that all the compounds except $\mathbf{5 f}, \mathbf{5 h}$, $\mathbf{5 l}$ and $\mathbf{5 p}$ exhibit moderate to good antioxidant properties. Among the analogs, compound $\mathbf{5 g}$ displayed best antioxidant property with $62.13 \%$ inhibition followed by $\mathbf{5 e}, \mathbf{5 k}$ and $\mathbf{5 c}$ with $55.48 \%, 49.34 \%$ and $42.41 \%$ activities, respectively.

In summary, a series of novel chromeno[4,3] pyridine-2,5-diones $\mathbf{5 a - p}$ were synthesized by a mild eco-friendly high yielding methodology using PEG as a recyclable solvent. The compounds were further subjected to antimicrobial and antioxidant screening where $\mathbf{5 d}$ and $\mathbf{5 k}$ displayed potent antimicrobial activities and compound $\mathbf{5 g}$ displayed promising antioxidant properties (table 4).

\section{Acknowledgements}

We thank the Management of Sankar Foundation for financial support and encouragement. The authors also thank SAIF, Cochin University of Science and Technology for elemental analysis.

\section{References}

1. (a) Jones G 1996 In Comprehensive heterocyclic chemistry II: A Mckillop (ed.) Pergamon Press, Oxford, 167; (b) Torres M, Gil S and Para M 2005 Curr. Org. Chem. 9 1757; (c) Cocco M T, Congiu C and Onnis V 2000 Eur. J. Med. Chem. 35 545; (d) Litvinov V P 2003 Russ. Chem. Rev. 72 69; (e) Murray $\mathrm{T}$ and Zimmerman S 1995 Tetrahedron Lett. 36 7627; (f) Guarna A, Belle C, Machetti F, Occhiato E G and Payne A H 1997 J. Med. Chem. 40 1112; (g) Li Q, Mitscher L A and Shen L L 2000 Med. Res. Rev. 20 231; (h) Stončius S, Orentas E, 
Butkus E, Öhrström L, Wendt O F and Wärnmark K 2006 J. Am. Chem. Soc. 128 8272; (i) Sasaki T, Guerrero J M, Leonard A D and Tour J M 2008 Nano Res. 1 412; (j) Mohler M L, Bohl C E, Jones A, Coss C C, Narayanan R, He Y, Hwang D J, Dalton J T and Miller D D 2009 J. Med. Chem. 523597

2. Richard T V 1864 J. Chem. Soc. 17195

3. (a) Elbein A D and Molyneux R J 1981 In Alkaloids Chemical Biological Perspectives, S Williams Pelletier (ed.) (John Wiley) 5 1; (b) Li Q, Mitscher L A and Shen L L 2000 Med. Res. Rev. 20 231; (c) Nagarajan M, Xiao X S, Antony S, Kohlhagen G, Pommier Y and Cushman M 2003 J. Med. Chem 465712

4. (a) Dolle, R E and Nicolaou K C 1985 J. Am. Chem. Soc. 107 1691; (b) Cox R J and Hagan D 1991 J. Chem. Soc. Perkin Trans. 12537

5. Gary D and Byron A K 1991 J. Ind. Microbiol. 8265

6. Presti E L, Boggia R, Feltrin A, Menozzi G, Dorigo P and Mosti L 1999 II Farmaco 54465

7. (a) Clercq, E D 1999 II Farmaco 54 26; (b) Parreira R L T, Abrahão O and Galembeck S E 2001 Tetrahedron 57 3243

8. Jessen H J and Gademann K 2010 Nat. Prod. Rep. 27 1168

9. Kaiser J P, Feng Y and Bollag J M 1996 Microbiol. Rev. 60483

10. Krawczyk S, Otto M, Otto A, Coburger C, Krug M, Seifert M, Tell V, Molnár J and Hilgeroth A 2011 Bioorg. Med. Chem. 196309

11. (a) Jang K H, Lee B H, Choi B W, Lee H S and Shin J 2005 J. Nat. Prod. 68 716; (b) Malquichagua S K J, Delgado P G E, Lluncor L R, Young M C M and Kato M J 2005 Phytochemistry 66 573; (c) Burkhardt G, Becker H, Grubert M T J and Eicher T 1994 Phytochemistry 37 1593; (d) Numata A, Kanbara S, Takahashi C, Fujiki R, Yoneda M, Usami Y and Fujita E 1992 Phytochemistry 31 1209; (e) Kulkarni M M, Nagasampagi B A, Deshpande S G and Sharma R N 1987 Phytochemistry 26 2969; (f) Isman M B and Proksch P 1985 Phytochemistry 24 1949; (g) Kitamura R O S, Romoff P, Young M C M, Kato M J and Lago J H G 2006 Phytochemistry 67 2398

12. (a) Shanthi G, Perumal P T, Rao U and Sehgal P K 2009 Indian J. Chem. Sec. B 48 1319; (b) Foroumadi A, Dehghan G, Samzadeh K A, Arabsorkhi F, Sorkhi M, Shafiee A and Abdollahi M 2007 Asian J. Chem. 19 1391

13. (a) Kumar D, Reddy V B, Sharad S, Dube U and Kapur S 2009 Eur. J. Med. Chem. 44 3805; (b) El-Saghier A M M, Naili M B, Rammash B K, Saleh N A and Kreddan K M 2007 Arkivoc. 1683

14. Conti C and Desideri N 2009 Bioorg. Med. Chem. 17 3720

15. Alizadeh B H, Ostad S N, Foroumadi A, Amini M, Dowlatabadi R, Navidpour L and Shafiee A 2008 Arkivoc $\mathbf{1 3} 45$
16. (a) Gourdeau H, Leblond L, Hamelin B, Desputeau C, Dong K, Kianicka I, Custeau D, Boudreau C, Geerts L, Cai S X, Drewe J, Labrecque D, Kasibhatla S and Tseng B 2004 Mol. Cancer Ther. 3 1375; (b) Reddy P. N, Reddy Y T, Rao M K and Rajitha B 2003 Heterocycl. Commun. 9647

17. Babu K S, Raju B C Praveen B, Kishore K H, Murty U S and Rao J M 2003 Heterocycl. Commun. 9 519; (b) ElGaby M S A, Zahran M A, Ismail M M F and Ammar Y A 2000 Farmaco 55227

18. Cassidy F, Evans J M, Hadley M S, Haladij A H, Leach P E and Stemp G 1992 J. Med. Chem. 351623

19. Maxwell A 1993 Mol. Microbiol. 9681

20. Soliman S G F and Kappe T Z 1976 Z. Naturforsch. B31 495

21. Heber D, Ivanov I C and Karagiosov S K $1995 \mathrm{~J}$. Heterocyclic. Chem. 32505

22. Ivanov I C, Glasnov T N and Heber D 2005 J. Heterocycl. Chem. 42857

23. Kafka S, Aigner R and Kappe T 2006 J. Heterocycl. Chem. 431105

24. (a) Suri O P, Satti, N K and Suri A 2000 Syn. commun. 30 3709; (b) Sirisha K, Bikshapathi D, Achaiah G and Reddy V M 2011 Eur. J. Med. Chem. 46 1564; (c) Virsodia V, Pissurlenkar R R, Manvar D, Dholakia C, Adlakha P, Shah A and Coutinho E C 2008 Eur. J. Med. Chem. 43 2103; (d) García M J, Rebolledo F and Gotor V 1993 Tertrahedron Lett. 34 6141; (e) Tennant G 1993 J. Chem. Soc. 2428; (f) García M J and Francisca R F 1994 Tetrahedron $\mathbf{5 0} 6935$

25. (a) Chandrasekhar S, Narsihmulu Ch, Sultana S S and Reddy N R 2002 Org. Lett. 4 4399; (b) Li J H, Liu W J and Xie Y X 2005 J. Org. Chem 70 5409; (c) Carlos K Z A and Luana M A 2005 Curr. Org. Chem. 9 195; (d) Ji C, Spear S K, Huddleston J G and Rogers R D 2005 Green Chem. 7 64; (e) Aires da Conceição S, Jaqueline D S, Aguiar L C S, Alessandro B C S, Andréa de Souza L F, Luiz F B M and Antunes O A C 2010 Tetrahedron Lett. 51 3883; (f) Gelson P, Elton L B, Paloma C R, Carvalho P N and Lenardão E J 2013 Tetrahedron Lett. 54 1718

26. (a) Prasad J V, Prabhakar M, Manjulatha K, Rambabu D, Solomon K A, Krishna G G and Kumar K A 2010 Tetrahedron Lett. 51 3109; (b) Prasad J V, Reddy J S, Kumar N R, Solomon K A and Gopikrishna G 2011 J. Chem. Sci. 123 673; (c) Jalli V P, Jaggavarapu S R, Kamalakaran A S, Gangisetty S K, Nanubolu, J B and Gopikrishna G 2013 Tetrahedron Lett. 54 1491; (d) Jaggavarapu S R, Kamalakaran A S, Gayatri, G, Shukla M, Dorai K and Gopikrishna G 2013 Tetrahedron 69 2142

27. O Halloran N, James J P, Downey C A, O' Malley P, Duff T and Bertrand S 2008 Heterocycles $\mathbf{7 5}$ 2681

28. Kim K S, Lee S, Lee Y S, Jung S H, Park Y, Shin K H and Kim B K 2003 J. Ethnolpharmacol. 8569 